\title{
High Spectral Resolution Atmospheric Emitted Radiance Studies with the ARM UAV
}

\section{Final Report}

To

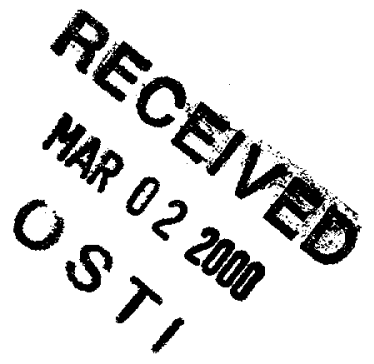

Patrick Crowley

DOE Project Officer, ER-74

U.S. Department of Energy

19901 Germantown Road

Germantown, MD 20874-1290

(301-903-3069)

For

DOE Grant \# DE-FG02-95ER61996

During

The Project Period

From: 03/01/1995

Thru: $02 / 28 / 1999$

From

Dr. Henry E. Revercomb

Principal Investigator

University of Wisconsin-Madison

Space Science and Engineering Center

1225 W. Dayton St.

Madison, WI 53706

(608-263-6758)

31 December 1999 


\section{DISCLAIMER}

This report was prepared as an account of work sponsored by an agency of the United States Government. Neither the United States Government nor any agency thereof, nor any of their employees, make any warranty, express or implied, or assumes any legal liability or responsibility for the accuracy, completeness, or usefulness of any information, apparatus, product, or process disclosed, or represents that its use would not infringe privately owned rights. Reference herein to any specific commercial product, process, or service by trade name, trademark, manufacturer, or otherwise does not necessarily constitute or imply its endorsement, recommendation, or favoring by the United States Government or any agency thereof. The views and opinions of authors expressed herein do not necessarily state or reflect those of the United States Government or any agency thereof. 


\section{DISCLAIMER}

Portions of this document may be illegible in electronic image products. Images are produced from the best available original document. 


\section{Table of Contents}

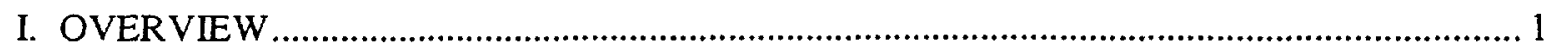

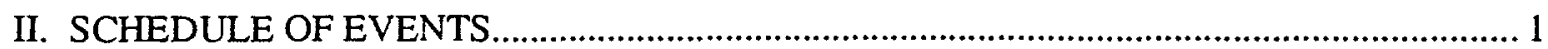

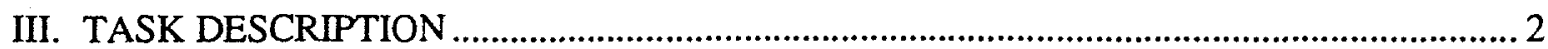

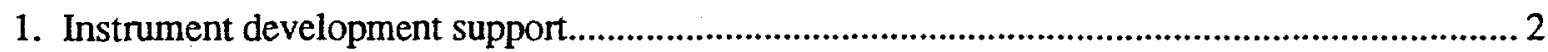

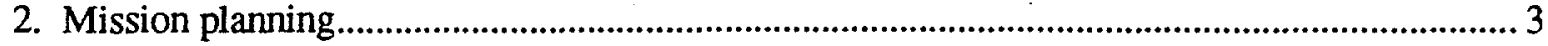

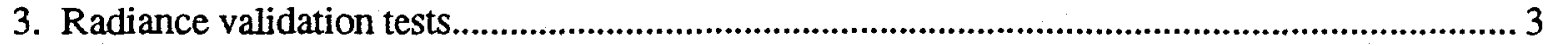

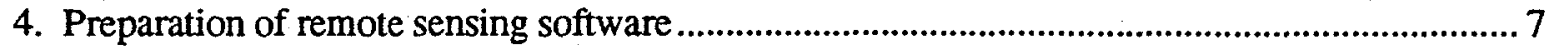

5. Technique development for analysis of pseudo in situ observations .................................... 7

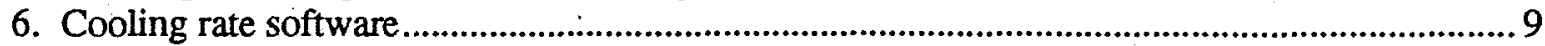

7. Coordinate software implementation into the ARM data system ..................................... 11

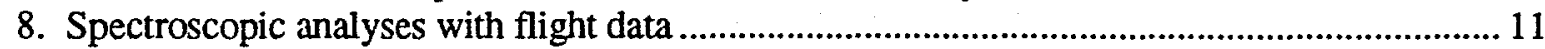

9. Upper level water vapor analyses with flight data ................................................................. 11

IV. SUMMARY

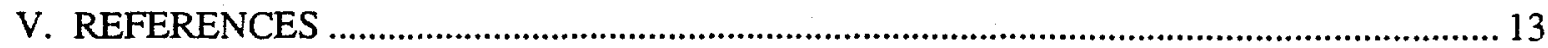

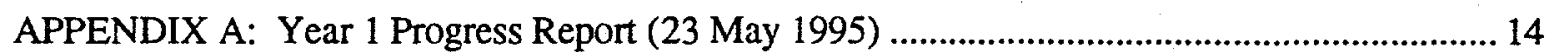

APPENDIX B: AERI-UAV Instrument Report (1996) ................................................... 15

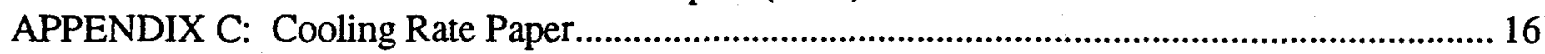




\section{OVERVIEW}

This is the final report of DOE Grant \# DE-FG02-95ER61996 to the University of Wisconsin Madison Space Science and Engineering Center for the project period from 03/01/1995 through 02/28/1999 titled "High Spectral Resolution Atmospheric Emitted Radiance Studies with the ARM UAV". The first year progress report of this grant is attached as Appendix A.

The intent of the original Department of Energy (DOE) grant study was to provide science team support for participation in the DOE Atmospheric Radiation Measurement (ARM) Unmanned Airbome Vehicle (UAV) program with a new radiation measurement instrument, the AERIUAV. At the time, the AERI-UAV was under development at the University of Wisconsin with separate DOE funding from the Strategic Environmental Research and Development Program (SERDP). Unfortunately, for programmatic reasons the development of the AERI-UAV was terminated before the integration of the AERI-UAV instrument with the intended UAV platform was completed and the scope of the ARM UAV program was scaled back to exclude the high spectral resolution radiation measurements.

The original goals of the AERI-UAV instrument development are described in the final report for that project which is attached as Appendix B. An extract of the final report (1996) on the original AERI-UAV instrument development at the University of Wisconsin is provided below:

"The Atmospheric Emitted Radiance Interferometer (AERI) for an Unmanned Aerospace Vehicle (UAV) is now completing development at the University of Wisconsin as part of the DOE Atmospheric Radiation Measurement (ARM) Program. The AERI-UAV makes broadband infrared (3-20 $\mu \mathrm{m}$ ), high spectral resolution $\left(0.3-0.5 \mathrm{~cm}^{-1}\right)$ observations of atmospheric emission, providing key meteorological information related to atmospheric state parameters (temperature, water vapor, and other greenhouse gases), cloud and surface spectral properties, and processes influencing radiative budgets and regional climate. The AERI-UAV uses state-of-the-art Fourier Transform Spectroscopy (FTS), or Michelson interferometry, which has proven to be an exceptionally effective approach for making these IR spectral observations with the high radiometric accuracy necessary for weather and climate applications"

Without DOE funding for UAV integration and flight testing, it was necessary to secure external funding to integrate the instrument on NASA research aircraft (the DC-8 and the ER-2). In the spirit of the original proposal, this report provides a summary of the current state of progress in the study of high spectral atmospheric emitted radiance from the instrument formerly known as the AERI-UAV but subsequently renamed the Scanning High-resolution Interferometer Sounder (S-HIS) and flown on several NASA missions. This document contains an overview, a schedule of events relevant to this grant, a task description following the outline of the original proposal, and a summary.

\section{II. . SCHEDULE OF EVENTS}

The following list details the important events occurring both within and external to this grant which have affected the conduct of the grant study.

- 1 March 1995: Start of DOE grant titled "High Spectral Resolution Atmospheric Emitted Radiance Studies with the ARM UAV"

- 1-3 March 1995: Science presentation of AERI-UAV at the First ARM-UAV Science Team Meeting, Dallas-Fort Worth. TX.

- 23 May 1995: Year one progress report submitted (see Appendix A). 
- Early 1996:

DOE funding for AERI-UAV instrument development is terminated before integration with ARM UAV is completed. Funding for operation of the AERI-UAV on the ARM UAV is not available for programmatic reasons.

- Early 1997: NASA and IPO (Integrated Project Office) funding is obtained to modify and fly the hardware components of the AERI-UAV on the NASA DC-8 and ER-2 research aircraft. The instrument in this new configuration is renamed the Scanning - High resolution Interferometer Sounder (S-HIS).

- February 1997: DOE grant study period is extended until 02/28/1999 in order to include examples of flight data analysis from the S-HIS instrument.

- September 1998: Scanning-HIS operates successfully in the NASA Convection and Moisture Experiment-III (CAMEX3) experiment with flights of the NASA DC-8 aircraft out of Patrick AFB in Florida.

- March 1999: - Scanning-HIS operates aboard the NASA ER-2 aircraft during the Winter Experiment (WINTEX 1999) based in Madison, Wisconsin.

- September 1999: Scanning-HIS participates in the NASA TRMM validation mission (KWAJEX) aboard the NASA DC-8 aircraft based at Kwajalein Atoll in the tropical western pacific.

- November 1999: Final report of this DOE grant completed.

- October 2000: . Proposed participation of S-HIS instrument (aboard NASA DC-8 aircraft) in the DOE ARM sponsored Water Vapor IOP at the Cloud and Radiation Testbed (CART) site in Oklahoma.

\section{TASK DESCRIPTION}

This report is organized according to the tasks outlined in the original proposal. The emphasis of the work performed under this grant has changed over the course of the study period in order to accommodate the changing of the observation platform from the UAV to the NASA aircraft platforms. The work performed under this grant fell under the original task categories and has been supplemented by other govemment contracts (mainly from NASA) which have also contributed to progress in these areas. The task descriptions presented here represent a partial summary of the current state of high spectral resolution remote sensing with a focus on the measurements made with the Scanning-HIS as flown on the DC-8 and ER-2 research aircraft. As appropriate, distinction is made in each task between results funded directly from this grant and those supported in part or totally under separate contract.

\section{Instrument development support}

A substantial portion of the grant budget was expended in the task of supporting the development of the high spectral resolution instrumentation needed for the performance of this grant. The development of software tools based on the existing AERI data stream made possible the analysis of the instrument performance characteristics from recent flights of the Scanning-HIS. In the end, 
the calibration algorithms developed for the ARM AERI instruments have been successfully modified for routine application to the Scanning-HIS instrument flight data.

\section{Mission planning}

After actively participating in the first ARM-UAV science team meeting and attendance at a subsequent workshop, the reorganization of the ARM-UAV program limited our involvement in subsequent ARM-UAV mission planning.

\section{Radiance validation tests}

The primary product of the high spectral resolution observations are the calibrated radiance measurements themselves. Great care has been taken to design an instrument that can be accurately calibrated both radiometrically and spectrally. The calibration philosophy has followed the approach of the groundbased AERI system whereby two onboard reference blackbody cavities (one heated and one at ambient temperature) are repeatedly viewed during the flight to account for instrument temperature drifts with time. The two point calibration is augmented with a nonlinearity correction for certain detectors and an instrument line shape adjustment for all wavelengths. Instrument intercomparisons have been successfully performed with both a groundbased AERI instrument and with another FTIR instrument in-flight.

Having established the calibration reference for the Scanning-HIS instrument it is possible to begin the process of validation of atmospheric state parameters using the infrared spectral observations. One example of this is shown in figures 1-3 from the recent KWAJEX field experiment in support of the NASA TRMM mission. Analysis of the KWAJEX data was performed under separate NASA funding. Figure 1 shows the flight track of the DC- 8 aircraft over and around the islands that make up the Kwajalein Atoll in Marshall Islands of the tropical Pacific. Radiosondes were launched from three islands near Kwajalein during a period where the aircraft flew in a pattem near each under clear sky conditions. Figure 2 shows an overlay of the three radiosondes launched on this day. Note that one of the radiosondes is much cooler and drier than the other two. Figure 3 shows a comparison of the three Scanning-HIS observations with a calculation using the ARM radiative transfer code LBLRTM (S. Clough, AER, Inc.) with input from one of the radiosonde profiles (from Roi island). The agreement between observation and calculation shown in figure 3 is relatively good. The good agreement in figure 3 for the Scanning-HIS longwave band is a confirmation of the observed temperature profile (Roi island) used in the calculation. In other spectral regions, significant issues remain for the validation of water vapor, both in the atmospheric column and at high altitudes, as discussed further in section 9.

Through these radiance investigations the Scanning-HIS will play a role analogous to the ARM surface based AERI instruments in accurately measuring the clear sky infrared emission to space and validating our ability to accurately represent that emission with calculations. We have in the past and continue to work with Tony Clough of AER, Inc. and Larrabee Strow of University of Maryland Baltimore County in the testing and refinement of line-by-line models. Having established the accuracy of clear sky calculations, results from analyses of cloud sky observations can be made with greater confidence. 

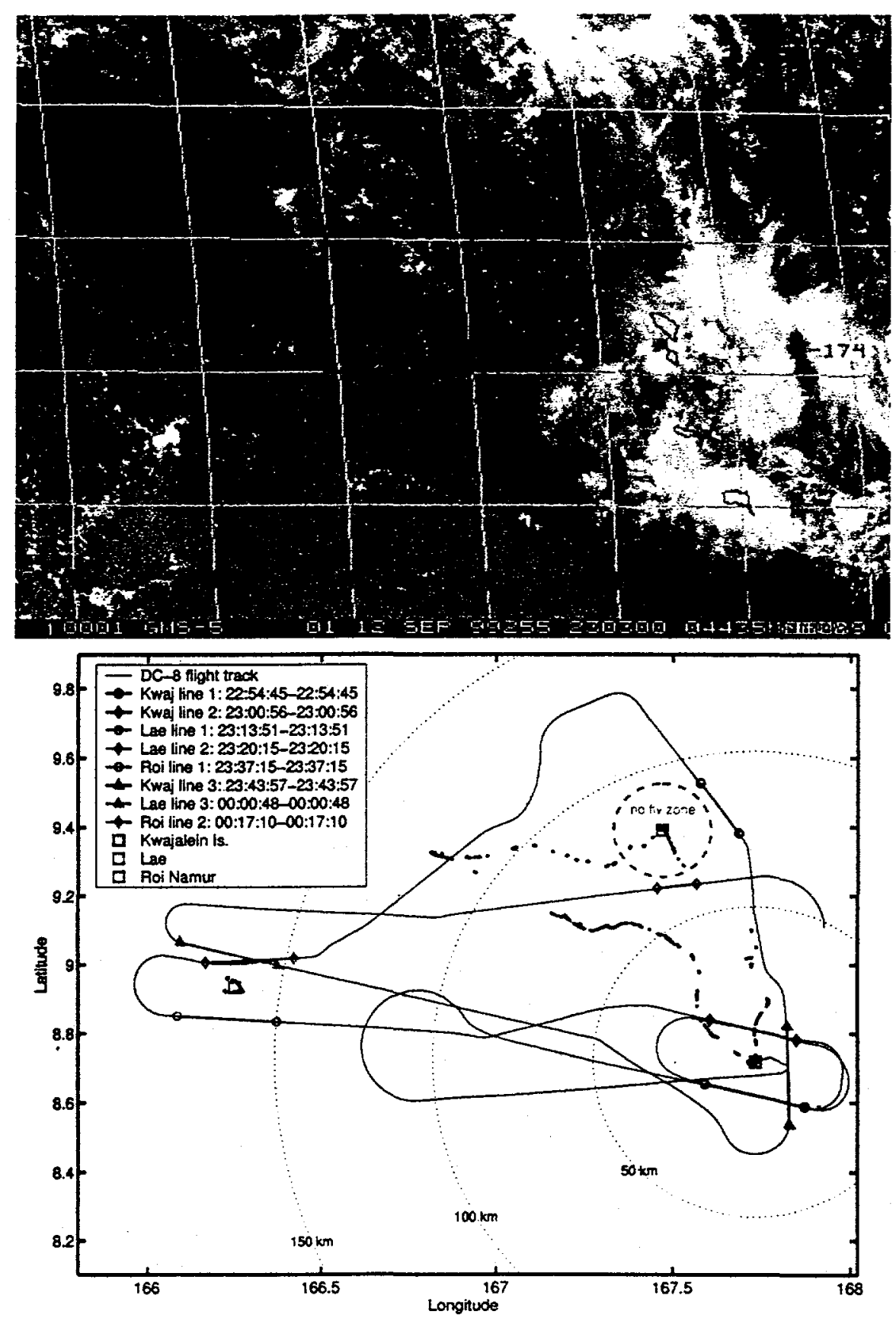

Figure 1. The highlighted region of the GMS satellite image in the upper panel is shown expanded in the lower panel to illustrate the flight track of the NASA DC-8 aircraft over the Kwajalein Atoll (dotted outline) during a dedicated "clear sky" Scanning HIS mission on 12 September 1999. 

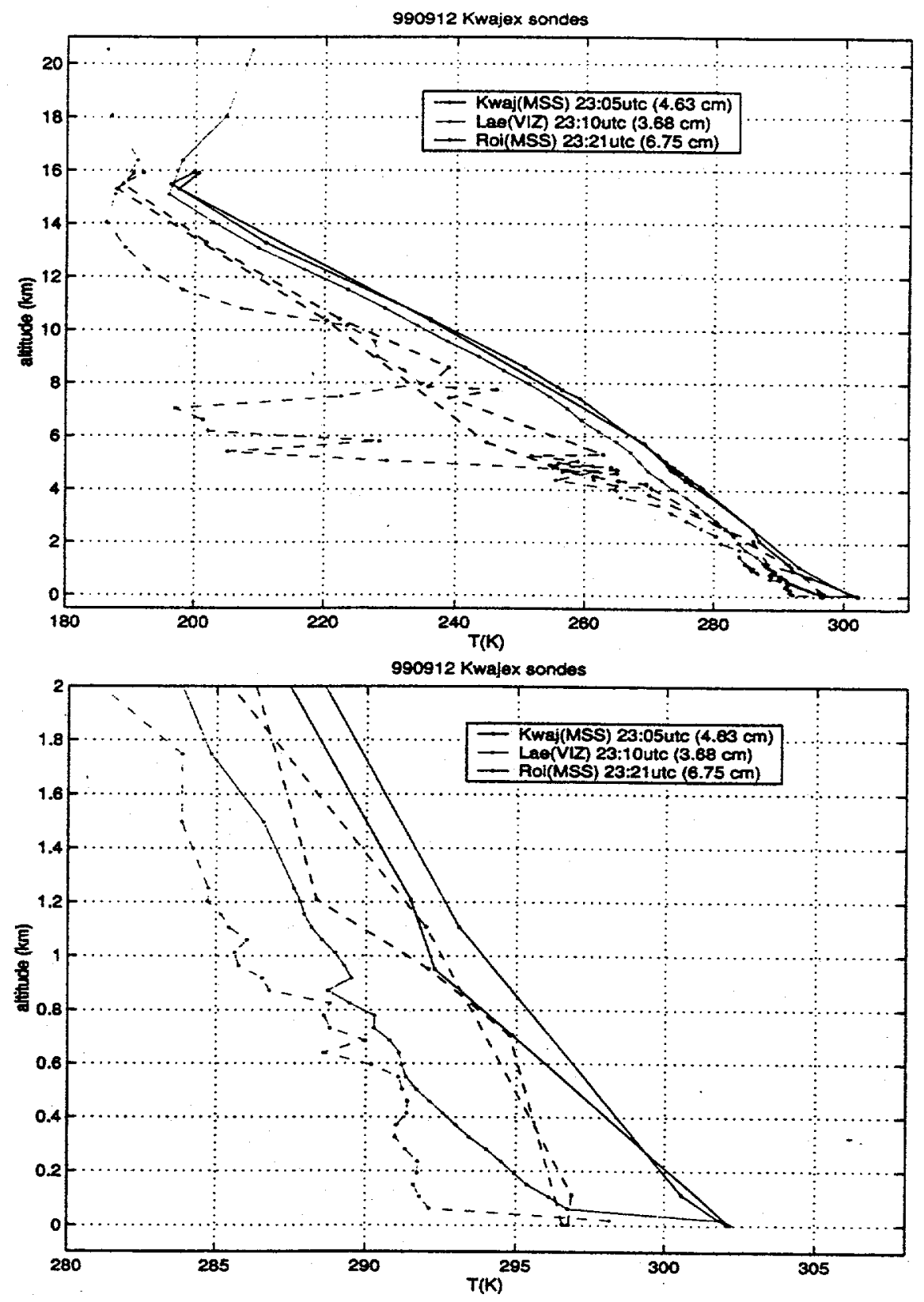

Figure 2. Overlay of radiosondes launched on 12 September 1999 from the islands of Kwajalein, Lae, and Roi in the Marshall Islands. Note that the radiosonde launched from Lae island differs significantly in both temperature and water vapor from those launched from Kwajalein and Roi. This is thought to be a sonde measurement error in the Lae sounding. 
990912 Rol overpasses
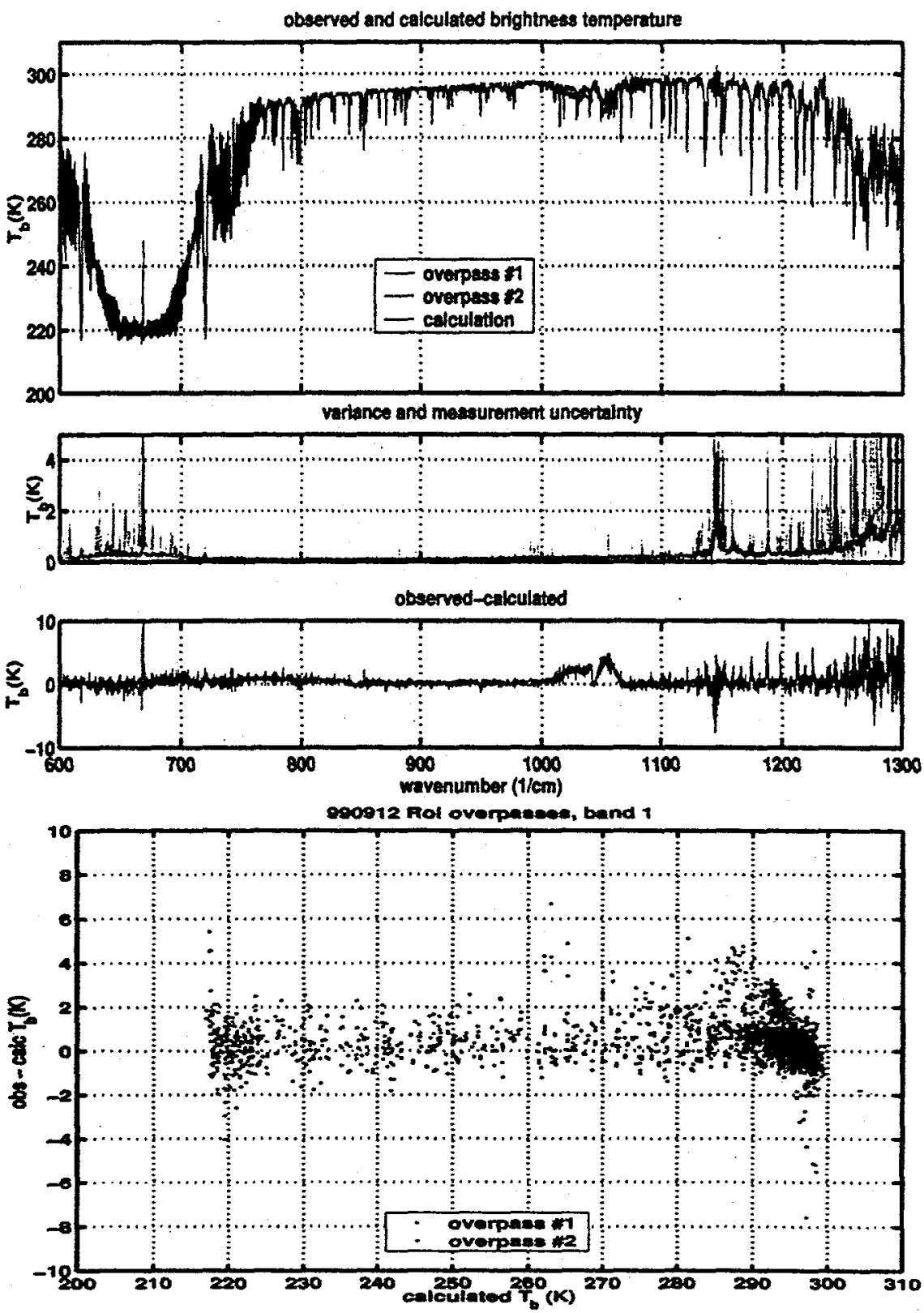

Figure 3. Clear sky comparison of Scanning-HIS brightness temperature observations (two overpasses) from the NASA DC-8 aircraft over Roi Island in the Tropical Western Pacific to a line-by-line calculation using a coincident radiosonde for the temperature and water vapor profile. 


\section{Preparation of remote sensing software}

A fast forward model has been developed for the Scanning-HIS instrument and the process of retrieval of the atmospheric state parameters (temperature, water vapor, and ozone) have begun under separate project grants. The radiance validation data sets indicated in task 3 will be used to validate the radiative transfer models used in the remote sensing analyses.

\section{Technique development for analysis of pseudo in situ observations}

The original proposal outlines the value of obtaining measurements from several altitudes in order to derive information about the emission from the layer bounded by the observations. Clear sky observations at several altitudes over a uniform scene have not yet be obtained. However, a good example of the value of such "pseudo in situ" observations was achieved during the recent NASA funded field experiment, KWAJEX. This result is described below.

High spectral resolution Scanning-HIS measurements, within the longwave atmospheric window, provide the capability to analyze upwelling radiation without contamination from ozone or strong water vapor absorption. This provides the potential for multi-spectral analysis of sea surface, land, vegetation, and cloud emissivity. Using observations from above and below an atmospheric feature (such as a cloud) it is possible to derive the characteristics of the feature which are important for its radiation. The KWAJEX field mission allowed us to measure upwelling atmospheric emission through cirrus clouds over a near-uniform ocean emission source. Multispectral analysis of cirrus clouds using high spectral resolution radiance data has been demonstrated from both ground- (DeSlover et al., 1999) and aircraft-based (Smith et al., 1998) measurements.

The first portion (near 0400 UTC) of the 26 August 1999 flight (DC8 sortie 990524) was dedicated to a cirrus mission while in transit to the TRMM satellite overpass. For this case the DC8 flew below the cloud, to measure the upwelling clear sky radiance; above the cloud, to measure the attenuated radiance through the cirrus cloud; and through the cloud, to measure both the emission at various cloud depths and the ice crystal size and habit with the 2D probe operated by Desert Research Institute (DRD). This case provided a unique opportunity to fly above and within cirrus clouds from the DC8 perspective, where the majority of cirrus during the KWAJEX mission was noted to be above the DC8 flight altitude. Figure 4 shows the radiance spectra from above and below a cirrus cloud and the derived spectral emissivity.

We anticipate that measurements of water vapor in clear air will also be possible using the "stair-step" method of observations at several altitudes. We look forward to the upcoming ARM Water Vapor IOP to collect the data needed to test these ideas. 

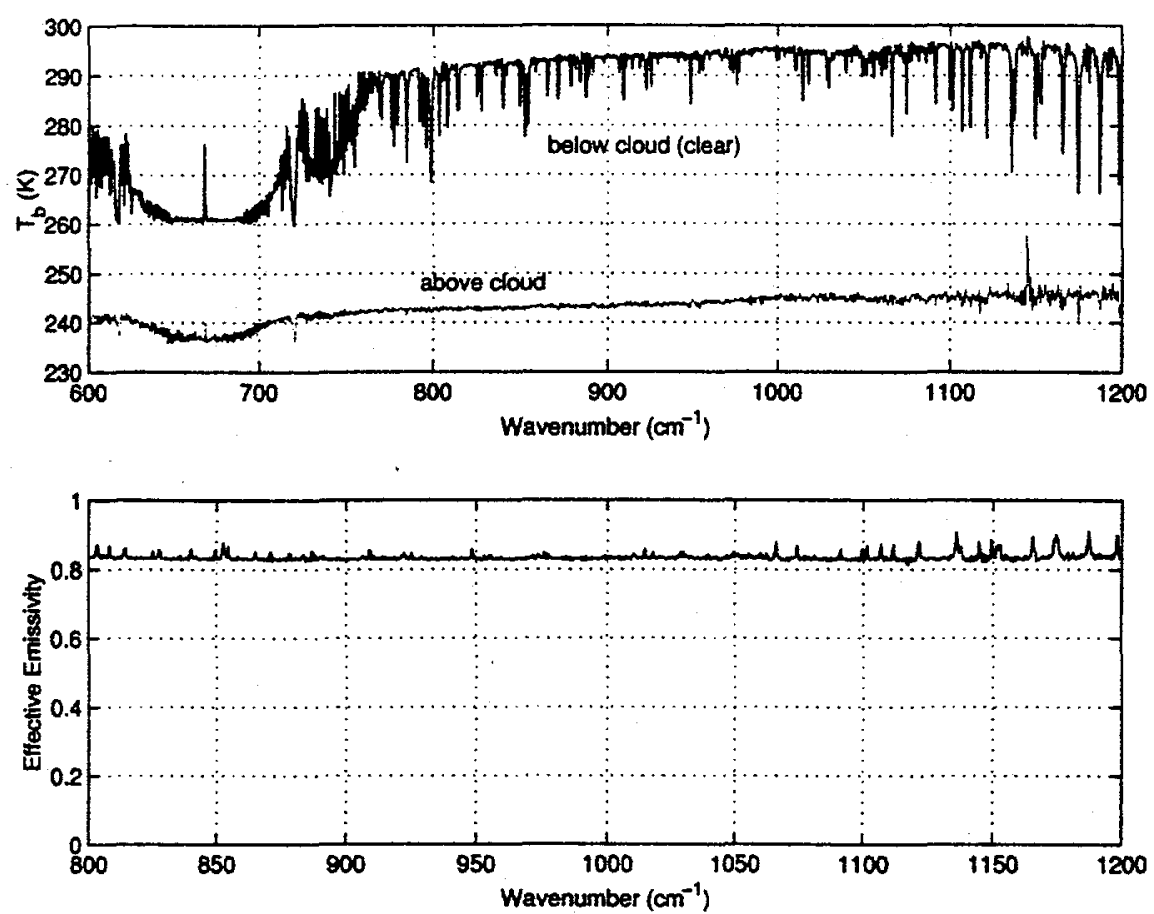

Figure 4. Scanning-HIS brightness temperature observations from the NASA DC-8 during the KWAJEX experiment from above and below a cirrus cloud deck (upper panel) and the derived effective emissivity (lower panel). The spectrally uniform cloud emissivity, near 0.83 across the longwave window, is indicative of ice particles with effective radius greater than 50 microns (Deslover et al., 1999). This is consistent with measurements from the $2 \mathrm{D}$ probe measurements of the DC-8 aircraft. 


\section{Cooling rate software}

The original proposal for generating spectral cooling rates for an atmospheric layer directly from the difference of the net spectral radiance at the top of the layer from that at the bottom remains unfinished due to lack of available observed data. However, an extensive software development and testing program was conducted under this grant for the implementation of software for calculating spectral fluxes and cooling rates in an accurate yet efficient manner. The approach taken was to build the spectral flux caculations around the version of the Phillips Laboratory software MODTRAN available at that time, MODTRAN3 (Anderson, et al., 1994). The SSEC model developed at Wisconsin for calculating spectral and cooling rates were validated against the published results of S. A. Clough (Clough, et al., 1992) based on careful line-by-line calculations. The result of this comparison is shown in table 1 and shows excellent agreement between the two models for the upwelling flux at the surface, the downwelling flux at the surface, and the upwelling flux at the top of the atmosphere. The MODTRAN based calculation model has a tremendous computational speed advantage over the line-by-line model while retaining the capability of computing spectral flux at relatively high spectral resolution.

Table 1. Comparison of the infrared fluxes from the cooling rate model (SSEC) developed at the Uni. of Wisconsin under this grant (based on Phillips Lab's MODTRAN) to S. A. Clough's lineby-line model cooling rate results (AER). The case compared is the mid-latitude summer reference atmosphere for $\mathrm{H}_{2} \mathrm{O}$ (including continuum), $\mathrm{CO}_{2}$, and $\mathrm{O}_{3}$ over the spectral range 10 $3000 \mathrm{~cm}^{-1}$. Units are $\mathrm{W} / \mathrm{m}^{2}$.

\begin{tabular}{|l|l|l|l|}
\hline MLS & AER & SSEC & Flux Difference \\
\hline SFC $\uparrow$ & 423.5 & 423.4 & $0.03 \%$ \\
\hline SFC $\downarrow$ & 346.9 & 347.5 & $0.17 \%$ \\
\hline TOA & 283.3 & 283.7 & $0.13 \%$ \\
\hline
\end{tabular}

The SSEC model for cooling rate calculations was used extensively in the analysis of the high spectral resolution observations collected over the antarctic polar vortex in 1994 from the NASA ER-2 during the Airbome Southern Hemisphere Ozone Experiment. One of the objectives of the ASHOE was to examine the causes of ozone loss in the lower stratosphere, and to investigate how the loss is related to polar, mid-latitude, and tropical processes, including radiative transfer. Cooling rates were calculated for ASHOE in order to: (1) understand how the ozone depleted air is transported to populated regions outside the polar vortex, (2) relate vertical motions and transport of air around the South Pole to atmospheric cooling rates, and (3) validate cooling rates in dynamical models for the polar vortex using observed data and more accurate radiative transfer models. Temperature and water vapor retrievals from the UW High-resolution Interferometer Sounder (HIS) flights during ASHOE were used to calculate cooling rates, which compared favorably with results using other global model inputs. Results of these case studies showed that dynamical models of the polar vortex tend to contain a drier than observed upper atmosphere in the arctic and this leads to a lower cooling rate in the upper troposphere than is implied by the HIS observations.

During the course of this grant study, S. A. Clough of AER, Inc. released a version of his Rapid Radiative Transfer Model (RRTM) developed with support of the DOE ARM program. We conducted a series of tests of the SSEC cooling rate model (based on MODTRAN) with the RRTM model. Figure 5 illustrates the excellent agreement obtained in the cooling rate vertical 


\section{Cooling Rate Comparison between Two Models for ASHOE - 3/4 October 68S}

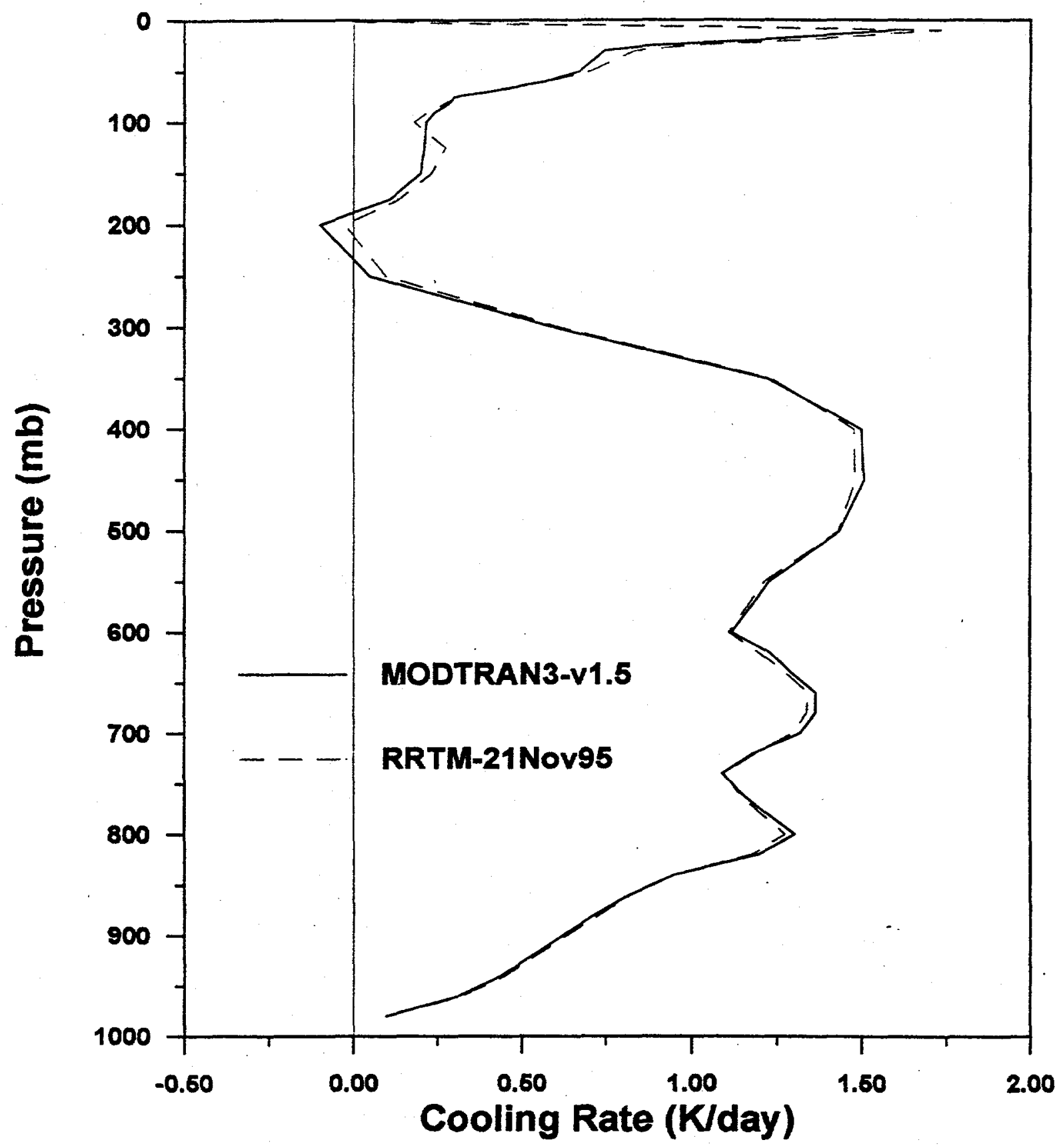

Figure 5. Comparison of MODTRAN3 derived cooling rate profile to that obtained from RRTM for the same input profile. The agreement is excellent. 
profiles obtained with these two models for an observation taken over the ozone hole (68 South latitude) from the ASHOE experiment. The SSEC model has the advantage over RRTM of being able to simulate higher spectral resolution fluxes of the type observed directly with the ScanningHIS. Results from these cooling rate investigations were reported in two conference papers (Lee. 1996; Lee, 1997).

\section{Coordinate software implementation into ARM data system}

The need for this task has not arisen since data of this type have not yet been taken in support of the ARM program. However, the data structures adopted for the Scanning-HIS (using the netCDF format) have evolved from the ARM AERI data steam and are expected to be highly compatible with the ARM data system. The first anticipated data set from Scanning-HIS to be provided to the ARM data system will be from the October 2000 Water Vapor IOP at the CART site in Oklahoma.

\section{Spectroscopic analyses with flight data}

The AERI/LBLRTM Quality Measurement Experiment (QME) of the DOE ARM program is the ground based analog for the comparison of observations and calculations that will be conducted using the aircraft data. These comparisons can be used for the improvement of infrared emission lines properties (positions, strengths, and widths) and continuum contributions (e.g. water vapor far wing effects). Future observations from the DOE ARM Third Water Vapor Intensive Operating Program (in October 2000) will provide a basis for these spectroscopic analyses in the context of the ARM program.

\section{Upper level water vapor analyses with flight data}

The accuracy of the upwelling radiance measurements combined with the sensitivity of emission to water vapor content in the upper troposphere make the Scanning-HIS measurements valuable for the evaluation of other more direct measurements of upper level water vapor. In particular, discrepancies between sonde measurements of water vapor in the upper troposphere have been observed for many years. By comparing Scanning-HIS observations to calculations performed with coincident radiosonde measurements we will be able to assess the accuracy of the in situ measurements from radiosondes.

An example of this comes from the recent NASA funded KWAJEX field experiment. The same case described in section 3 previously (see figures 1-3) can be studied with regard to upper level water vapor. The Scanning-HIS "band 2" measurements cover the 6.3 micron water vapor band which is sensitive to altitudes including the upper troposphere. Figure 6 shows a comparison of the water vapor band comparisons between Scanning-HIS observations and calculations using radiosondes as input. Clearly the comparison with the Roi radiosonde is much better (average error is closer to zero) than the comparison with the Lae radiosonde. Recall that the Lae radiosonde profile is one shown in figure 2 which is cooler in temperature and drier in water vapor content than the other two profiles (from Kwajalein and Roi). From the comparison with the Scanning-HIS observations we can infer that the Lae radiosonde profile is significantly in error.

Further analyses of this are anticipated in direct support of the DOE ARM program during the October 2000 Water Vapor IOP at the Oklahoma CART site. 

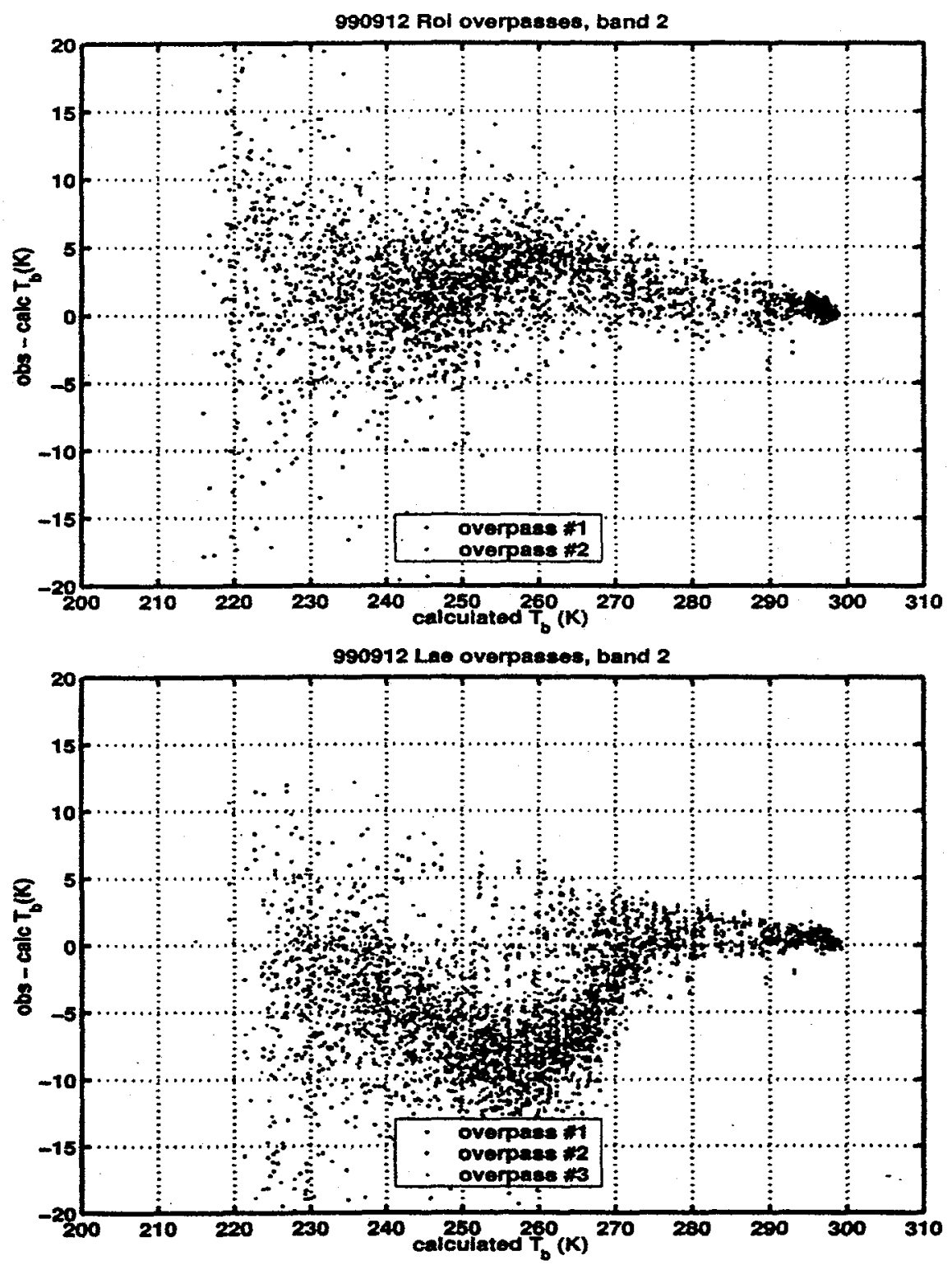

Figure 6. The Scanning-HIS observations in the 6.3 micron water vapor band made at high spectral resolution are shown here as a brightness temperature difference from a calculation performed using radiosondes launched at Roi and nearby Lae island on 12 September 1999 in the tropical western pecific. The upper figure is the comparison of Scanning-HIS with the radiosonde launched at Roi and shows generally good average agreement through the water vapor band. The lower figure is the comparison with the Lae radiosonde which confirms a problem with the water vapor measurements from that radiosonde. 


\section{SUMMARY}

The active participation in the ARM UAV science team that was anticipated in the grant proposal was indefinitely delayed after the first year due to a programmatic decision to exclude the high spectral resolution observations from the existing ARM UAV program. However, this report shows that substantial progress toward the science objectives of this grant have made with the help of separate funding from NASA and other agencies. In the four year grant period (including time extensions), a new high spectral resolution instrument has been flown and has successfully demonstrated the ability to obtain measurements of the type needed in the conduct of this grant. In the near term, the third water vapor intensive observing period (WVIOP-3) in October 2000 will provide an opportunity to bring the high spectral resolution observations of upwelling radiance into the ARM program to complement the downwelling radiance observations from the existing ARM AERI instruments. We look forward to a time when the ARM-UAV program is able to extend its scope to include the capability for making these high spectral resolution measurements from a UAV platform.

\section{REFERENCES:}

Anderson, G.P., J. H. Chetwynd, F.X. Kneizys, L.A. Hall, L.M. Kimball, 1994: MODTRAN3: Suitability as a Flux-Divergence Code, personal communication.

Clough, S. A., M. J. Iacono, and J.-L. Moncet (1992): Line-by-line calculation of atmospheric fluxes and cooling rates: Application to water vapor, J. Geophys. Res., 97, 15,761-15785.

DeSlover, D. H., W. L. Smith, P. K. Piironen, and E. W. Eloranta (1999): A methodology for measuring cirrus cloud visible to infrared optical depth ratios. J. Atmos. Oceanic Technol., 16, pp. $251--262$.

Lee, Szu-Chia, H. Revercomb, R. Knuteson, and W. Smith (1996): Cooling Rate Calculations for the Airborne Southern Hemisphere Ozone Experiment (ASHOE) using MODTRAN3, HITRAN Review Conference,

Lee, Szu-Chia, R. Knuteson, H. Revercomb, and W. Smith (1997): Atmospheric Cooling Rate Calculations for the Airbome Southern Hemisphere Ozone Experiment (ASHOE) using MODTRAN3, Optical Remote Sensing of the Atmosphere, Winter Topical Meeting 1997. Optical Society of America.

Smith, W. L., S. Ackerman, H. Revercomb, H. Huang, D. H. DeSlover, W. Feltz, L. Gumley, and A. Collard (1998): Infrared spectral absorption of nearly invisible cirrus clouds. Geophys. Res. Lett.. 25, pp. 1137-1140. 
APPENDIX A: $\quad$ Year 1 Progress Report (23 May 1995) 
Progress Report on the ARM-UAV Grant:

High Spectral Resolution Atmospheric Emitted Radiance Studies with the ARM UAV

This first year of the grant to develop analysis techniques and observing strategies for the effective use of the new Atmospheric Emitted Radiance Interferometer (AERI-UAV) began with a very successful Science Team Meeting and several of the tasks under the grant are now underway. The separate instrument development effort is making good progress (documentation from the first design review provides a complete description of the concepts to be implemented). and we are very enthusiastic about the possibilities offered by the AERI-UAV to provide major improvements in our understanding of the effects of upper level water vapor on outgoing longwave radiation and on cloud formation. We look forward to the end of 1996 when extended flights of the ARM UAV over the CART site are planned.

As proposed, during the first 18 months before flight data is available, the planned tasks under the grant provide analysis support for instrument development, mission and flight planning to prepare for effective use of the new AERI-UAV, and development of techniques and software to take advantage of the unique UAV observing capabilities in the troposphere. These latter activities continue throughout the three year duration, as flight data becomes available for testing and science analyses.

The current status of each of the proposed tasks (except Tasks 8 and 9 for spectroscopic and upper level water vapor analyses that require flight data) is listed below:

Instrument development support

The portion of this task which is underway is the software development to enhance capabilities for diagnostic evaluation of instrument performance, including easy access to and display of phase characteristics, responsivities, noise performance, and housekeeping data. The similarity of the AERI-UAV data stream to that of the AERI makes it possible to make progress in this task even before data from the new instrument becomes available. We are making efforts to move toward the NETCDF data format to make the data self documenting and easily handled by users.

\section{(2) Mission planning}

We participated in the first ARM-UAV Science Team Meeting during which more detailed plans for the science rationale, choice of location, duration, instrument complement, and flight paths for future UAV missions were defined. An important consideration specific to the AERI-UAV will be the definition of the appropriate use of constant altitude observing and multiple altitude steps for pseudo in situ observing. Also, recent results from combined observations of the ground-based AERI and current University of Wisconsin aircraft instrument the HIS (High-resolution Interferometer Sounder) onboard the NASA ER2 were presented. The observations led to new techniques for analyzing sea surface temperature and emissivity that are directly applicable to data from the AERI-UAV for this project. 
While this task requires data from ground based tests of the instrument and has not been started. the software efforts being conducted under Task (1) will provide the basic techniques to facilitate routine performance evaluation and validation when the instrument tests begin.

\section{Preparation of remote sensing software}

Remote sensing from high resolution spectra fundamentally depends on having an accurate and rapid radiative transfer model to formulate the inverse solution for deriving atmospheric state parameters. The fast model currently used for HIS and AERI analyses is not as accurate as it can be. Therefore, we have initiated an effort to improve the fast model for use with the AERI-UAV software for temperature, water vapor, and ozone retrieval. A natural outgrowth of this effort will be a new, more accurate method for calculating the atmospheric weighting functions needed for retrieval. This effort will also include refinements of current techniques to allow the fast model to be used at the full resolution of the measurements (i.e. no apodization).

Future fast model efforts will address the issue of providing the capability for the fast model to deal with the wide range of altitudes over which the UAV can operate.

\section{Technique development for analysis of pseudo in situ observations}

The basic idea of the pseudo in situ observations from the AERI-UAV is that atmospheric absorption and gaseous concentrations can be derived more directly using observations of upwelling and downwelling radiances at two different altitudes, than from remote sensing with upwelling observations observed at a single altitude. However, the best way to formulate the mathematical approach to this analyis is not totally clear. We have derived equations that are valid using some assumptions and have performed simulations to define the associated errors. We have found the errors to be acceptably small over much of the important rotational water band region, that controls much of the earth cooling to space. However, further refinements are needed to treat other important regions.

\section{Cooling rate software}

The basis for generating spectral cooling rates for an atmospheric layer directly from the difference of the net spectral radiance at the top of the layer from that at the bottom is the conversion of radiance to flux. We have begun to study the angular dependence of longwave radiances in order to develop the software needed to convert AERI-UAV radiances to spectral fluxes. In addition, we have been investigating techniques for calculating atmospheric cooling rates from models using Modtran (Phillips Lab radiative transfer software) and other software.

\section{Coordinate software implementation into ARM data system}

The form of the current upgrades to software for the operational AERI-01 are well suited to the AERI-UAV data system needs and will make a significant contribution to this task.

The upcoming year of the program will be quite exciting as the AERI-UAV instrument comes together, and we expect that it will add a great deal to the already significant accomplishments of this young UAV program. We are convinced that the observations planned for the ARM-UAV are very important to the whole ARM Program, and hope that a way can be found to reap the scientific benefits of the program over the next decade. 
APPENDIX B:

AERI-UAV Instrument Report (1996) 


\title{
Atmospheric Emitted Radiance Interferometer (AERI)
}

\author{
for an Unmanned Aerospace Vehicle (UAV)
}

Developed for the

DOE Atmospheric Radiation Measurement (ARM) Program

\section{BACKGROUND AND SCIENTIFIC RATIONALE}

The Atmospheric Emitted Radiance Interferometer (AERI) for an Unmanned Aerospace Vehicle (UAV) is now completing development at the University of Wisconsin as part of the DOE Atmospheric Radiation Measurement (ARM) Program. The AERI-UAV makes broadband infrared (3-20 $\mu \mathrm{m})$, high spectral resolution (0.3$0.5 \mathrm{~cm}^{-1}$ ) observations of atmospheric emission, providing key meteorological information related to atmospheric state parameters (temperature, water vapor, and other greenhouse gases), cloud and surface spectral properties, and processes influencing radiative budgets and regional climate. The AERI-UAV uses state-of-the-art Fourier Transform Spectroscopy (FTS), or Michelson interferometry, which has proven to be an exceptionally effective approach for making these IR spectral observations with the high radiometric accuracy necessary for weather and climate applications. Two major advantages of an FTS design that have been recognized for several important applications are (1) the small instrument size, and (2) the high spectral integrity inherent in the FTS laser reference approach.

The AERI-UAV builds on other systems built at the UW including the NASA/NOAA High-resolution Interferometer Sounder (HIS) that flies on the NASA ER2 high altitude aircraft and the Atmospheric Emitted Radiance Interferometer (AERI) which was developed to make ground based measurements at the ARM Cloud and Radiation Testbed (CART) sites. The HIS has flown in almost 100 successful flights since 1985, including participation in eight major field programs in the last 6 years. The most recent campaign was the NASA SUCCESS (SUbsonic aircraft: Contrail and Cloud Effects Special Study) mission conducted over the ARM site in Oklahoma where the first operational AERI is located. The AERI has been providing a constant flow of radiometrically and spectrally calibrated data to the ARM science team via ethemet connections for over a year. Several additional AERI's are now being produced for deployment in the Tropical Western Pacific and the North Slope of Alaska, as well as extended sites in Oklahoma.

The AERI-UAV observations, like those of the HIS and AERI, are useful for addressing a wide range of science issues in addition to the remote sensing of temperature and water vapor that motivated our initial high resolution observations. Some of the important new capabilities of the AERI-UAV stem from its capability to observe both upwelling and downwelling radiance. The general nature of these observations is illustrated in Figure 1 by calculated upwelling and downwelling spectra at the $500 \mathrm{mb}$ level in the US Standard Atmosphere for the 7-20 $\mu \mathrm{m}$ region (full coverage includes 3.3-24 $\mu \mathrm{m}$ ). The higher radiance upwelling spectrum is bounded by the Planck radiances for the surface temperature and the $500 \mathrm{mb}$ temperature and the downwelling spectrum by the Planck radiance for the $500 \mathrm{mb}$ temperature and zero. The variable opacity of the greenhouse gases is apparent from the structure of the individual absorption lines, with the radiance of the more opaque regions being determined by the local atmospheric temperatures.

As shown from ground-based AERI observations, the more opaque regions dominated by $\mathrm{CO}_{2}$ absorption provide accurate temperature structure details, with especially high vertical resolution for altitudes within $1-2 \mathrm{~km}$ of the aircraft altitude. In fact, the shape of the spectra about the center of the $15 \mu \mathrm{m} \mathrm{CO} 2$ band $\left(620-720 \mathrm{~cm}^{-1}\right)$ varies markedly depending on whether the local temperature profile has a normal lapse rate or is inverted, and therefore, the radiance is not al ways bounded by the radiance corresponding to the local temperature as in Figure 1 . With this temperature information, the difference of spectra observed from two nearby altitudes gives accurate measures of the absorption (or transmission) of the intervening layer. 


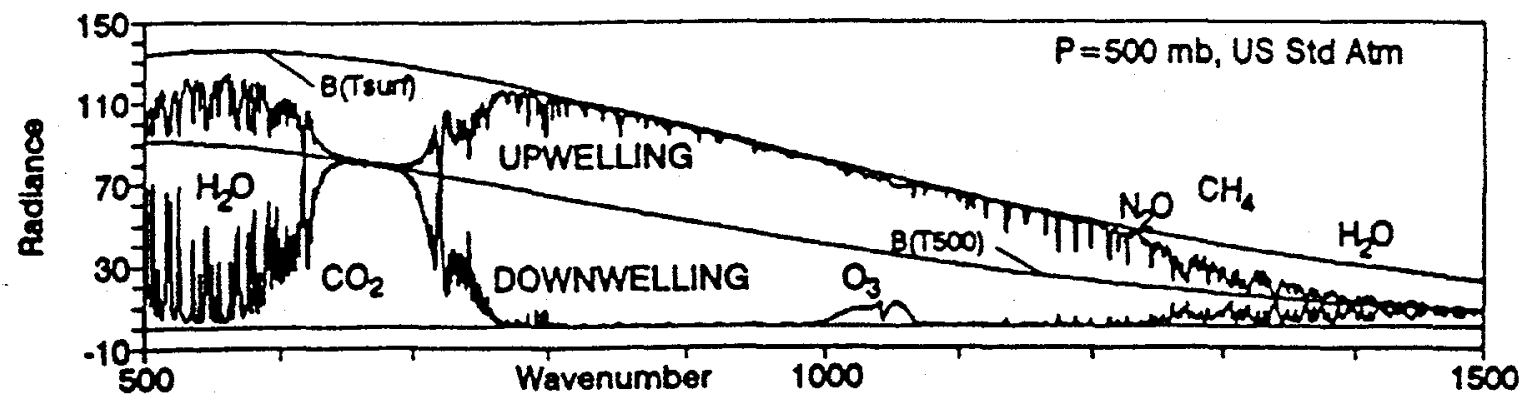

Figure 1. Simulation of partial AERI-UAV spectra from 7 to 20 microns.

A summary of some key science applications of the AERI-UAV is given in Table 1, along with a list of the specific products which can be derived from the fundamental calibrated radiance product. The first on the list, atmospheric spectroscopy, is a direct application of the radiances which analyses of HIS and AERI dan have proven to be very fruitful for improving radiative transfer models of the atmosphere. Both ground-based and airbome observations are needed to address some important spectroscopic issues over all of the relevant spectral regions.

Table 1. AERI-UAV Science Applications and Data Products

\begin{tabular}{|ll|}
\hline - Sclence Appllcatlons & - Products \\
\hline 1. More Sensitive Testing of Atmospheric & 1. State Parameters from Remote Sensing \\
Spectroscopy & 2. More Accurate Water Vapor and Stability \\
2. Improve Parameterizations of Cloud & 3. Radiative Cooling Rates" \\
Microphysics and Radiative Transfer & 4. Cloud Extinction and Optical Depth Spectra* \\
3. Observe Greenhouse Contribution from & 5. Profiles of Cloud Microphysical Propertiese \\
the Radiatively Active Trace Gases & 6. Absorption Coefficent Profiles* \\
4. Study the Spectral Radiative Forcing & 7. Trace Gas Mixing Ratio Profiles" \\
of Dynamical Processes & 8. Surface Spectral Emissivity \\
-Accuracy Enhanced from in situ measurements of vertical ofvergence of radiances
\end{tabular}

Cloud radiative transfer studies are one of the most fundamental applications of the AERI-UAV data for climate in conjunction with the ARM ground-based observations. Even without considering the inverse problem of obtaining cloud properties, comparisons of forward model radiances with any improved cloud radiative transfer schemes for models need to pass the test of statistically reasonable agreement with observed radiancer it is clear that the UAV will add new dimensions by offering cloud top information simultaneously with the cloud bottom information of AERI, and also the pseudo in situ profiling data for semi transparent clouds.

The observations of greenhouse contributions from radiatively active trace gases is a potentianly broad application deriving from the inherent sensitivity of longwave radiances to greenhouse gas concentrations. An especially important example is upper level water vapor which along with clouds has a large influence on the atmospheric energy balance. Ozone and CO retrieval are other areas of interest which parallel current activities with HIS and AERI data. The final application in Table I related to the spectral radiative forcing of dynamical processes is important for assessing the importance of spectrally varying properties on model results.

Previous experience with the HIS aircraft instrument has demonstrated that the basic products needed to support these science applications can be produced by remote sensing from observations at a constant altitude. However, a key feature of the UAV instrument is that many of these remote sensing products will be cahanced by 
the ability of the UAV to fly profiles in the troposphere. Observations at several altitudes inside the atmosphere will provide pseudo in situ information on atmospheric absorption and on local temperature and trace gas distributions. These pseudo in situ observations of atmospheric temperature and absorption are much more direct information than is usually obtained from remote sensing at a fixed altitude.

To summarize, in addition to providing the same wide range of spectral radiance and remote sensing information as that provided from high altitude by the HIS and from the ground by AERI, the AERI-UAV will give spectral cooling rates and effectively in situ cloud and state parameter information from upwelling and downwelling radiances acquired by flying constant-altitude steps.

\section{AERI-UAV INSTRUMENT DESIGN}

The fundamental AERI-UAV design was driven by the need to minimize size, weight and power because of the limited payload capacities of unmanned aircrat. In addition to substantially reducing the size compared to HIS and AERI, the requirements for the AERJ-UAV include maintaining the high radiometric and spectral calibration accuracy of the HIS and AERI instruments, while at the same time providing a facility instrument that is easy to operate and maintain. These latter factors led to a design that includes the fundamental FTS approach with accurate laser controlled sampling and includes a mechanical detector cooler to eliminate the need for liquid cryogens. The novel aspect of the design, a "4-color" shared focal plane detector configuration described in more detail below, followed from the size and power constraints; this configuration eliminates the need for dichroic beamsplitting and multiple coolers.

The general characteristics of the AERI-UAV are provided in Table 2 and the layout of the instrument is shown in Figures 2 and 3 . The $45^{\circ}$ scene miror rotates to select views of the atmosphere or of calibration reference sources (one near ambient temperature and one at a stabilized temperature up to $60^{\circ} \mathrm{C}$ ). A typical operational plan when flying at fixed altitudes in the troposphere is to alternate nadir and zenith viewing, with calibration cycles interspersed periodically.

Table 2. AERI-UAV Instrument Specifications

\begin{tabular}{|c|c|}
\hline Spatial Resolution & 100 mrad Nadir, Zenith, \& intermediate angles \\
\hline Spectral Resolution & $0.5 \mathrm{~cm}^{-1}$ \\
\hline Interforometer Type & $\begin{array}{l}\text { Voice Coil Dynamically Aligned Plane Miror } \\
\text { (Custom Bomem DA-5) }\end{array}$ \\
\hline Optlcal Path Drivel & Flex Pivot Porch Swing; $\pm 1.037 \mathrm{~cm} ; 4 \mathrm{~cm} / \mathrm{s}$ \\
\hline OPD Sampllng Reference & HeNe Laser with; white light at startup \\
\hline Fringe Counting & 1/4-wave quadrature, continuous backforth \\
\hline Interferometer Beam dlameter & $4.5 \mathrm{~cm}$ \\
\hline Interferometer Angular FOV (full) & $40 \mathrm{mrad}$ \\
\hline Spectral Bands & $\begin{array}{l}\text { Extra LW: } 19-25 \mu \mathrm{m} \\
\text { LW: } 10-19 \mu \mathrm{m} \\
\text { MW: } 5.5-10 \mu \mathrm{m} \\
\text { SW: } 3.3-5.5 \mu \mathrm{m}\end{array}$ \\
\hline Dotectors & InSb over 3-Segment MCT Array \\
\hline Cooler / Temperature & 0.6 W Stirting Cooler (Litton); $68 \mathrm{~K}$ \\
\hline Dwell TIme / Interferograms & 4 sec; 4 fore/ 4 back \\
\hline Onboard Processing & DSP Numerical Fiters \\
\hline Raw Data Rate & 80 Kbps \\
\hline Mase / Slze & $45 \mathrm{Kg} ; 0.05 \mathrm{~m}^{2}$ \\
\hline Power & $160 W$ \\
\hline
\end{tabular}


The fore-optics consists of a relatively compact 4-element afocal optics used to expand the beam and to convert the $100 \mathrm{~ms}$ angular field-of-view (FOV) for scene viewing 10 a $40 \mathrm{mr}$ interferometer FOV. This choice keeps the self-apodization reasonably small to minimize line shape uncertainties, but is larger than that used for HIS and AERI to improve noise performance. The interferometer is a highly customized version of the dynamically aligned Bomem DA-S. It was chosen over the Bomem comer cube design of the ground-based AERI because of its larger throughput and for the extra margin to environmental stresses offered by dynamic alignmeat.

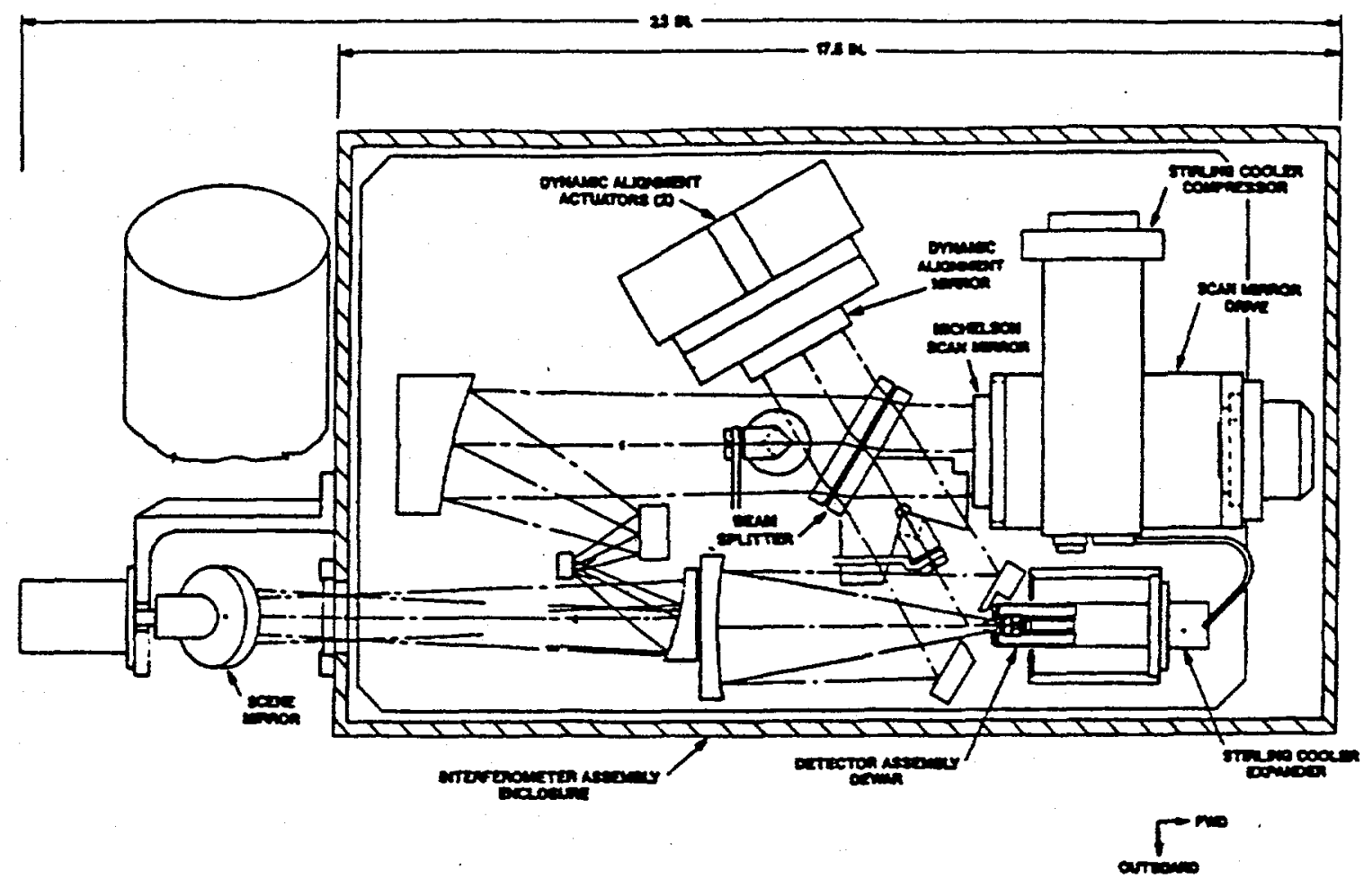

Figure 2. AERI-UAV top view assembly diagram, illustrating IR beam optical desigo with laser pick-off mirrors.

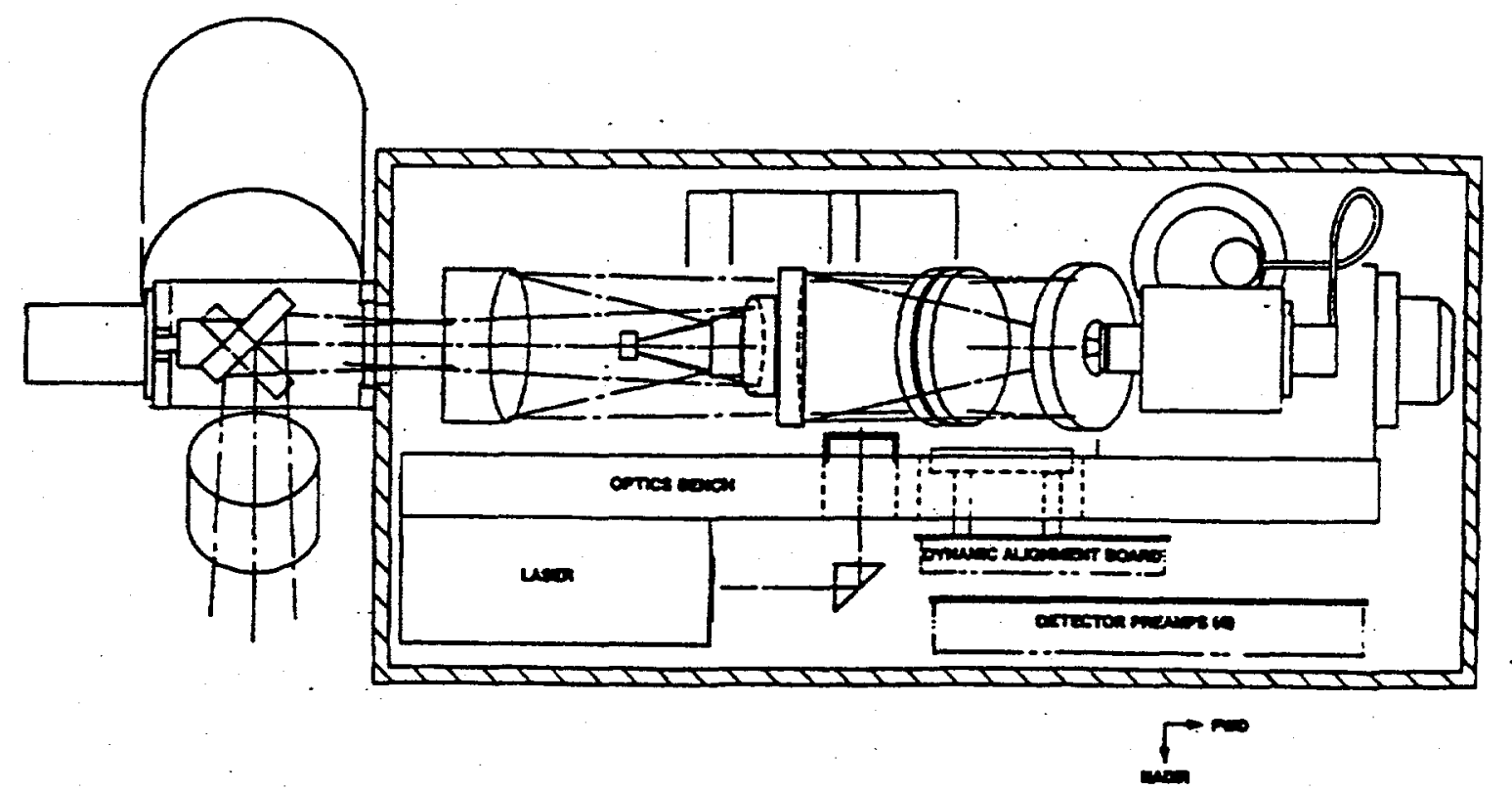

Figure 3. AERI-UAV side view assembly diagram, illustrating how the laser metrology system (mounted to the bottom of the optical bench) interfaces to the interferometer assembly on the top of the optical bench. 
The dynamic alignment mechanism (which maintains accurate optical alignment by tilting the "fixed" Michelson mirzor to compensate for tilts of the moving mirror using optical feedback signals derived from the expanded laser reference beam) uses a new voice coil actuator that is about 5 times faster than the standard mechanism on the HIS. The moving miror mechanism is also new. Its flex pivot design with voice coil actuation provides the required 4 $\mathrm{cm} / \mathrm{s}$ Optical Path Difference (OPD) scan rate. The more rapid interferogram scan rates of the AERI-UAV compared to the HIS allow lower altitude sampling without encountering large scene smear problems.

Another novel aspect of the AERI-UAV interferometer compared to the HIS is that it uses a white light source to identify an absolute ZPD fringe reference at startup and continuously counts fringes (forward and back, throughout turnarounds). The system is the same 1/4-wave quadrature system used in the AERI MB-100. This system allows onboard co-addition of interferograms to control data volume, while allowing rapid scanning to reduce FOV smear.

The aft optics, like the fore optics, is a compact design making use of a plane mirror and a single paraboloid outside of the detector dewar assembly. The IR beam from the paraboloid is focused through a concentric bole in the plane mirror (corresponding to the central obscuration of the interferometer sperture stop which accommodates the expanded laser reference beam). The field stop is mounted on the froat of the detector dewar assembly shown in Figure 4. Note that the dewar window is tilted significantly to prevent reflected radiation from double-passing the interferometer. The dewar contains two $\mathrm{KBr}$ lenses to image the aperture stop in the interferometer on the detectors.

The approach to spectral band separation for noise optimization is to share the beam aperture at the detector plane, as illustrated in Figure 5. This technique is not quite as efficient as using good dichroic beam splitters, but it is much more compact and provides a simple interface to a single, low-cost Stirling cooler. Also, it is much more efficient than the inexpensive, non-dichroic beamsplitters used inside the HIIS LHe dewar. The Stirling cooler is a $0.6 \mathrm{~W}$ Litton design with separate compressor and regenerator connected by a small gauge He filled tube. A major advantage of this cooler, which was chosen for our ground-based AERI instruments, is that it can be removed for refurbishment without disturbing the detector-dewar assembly.
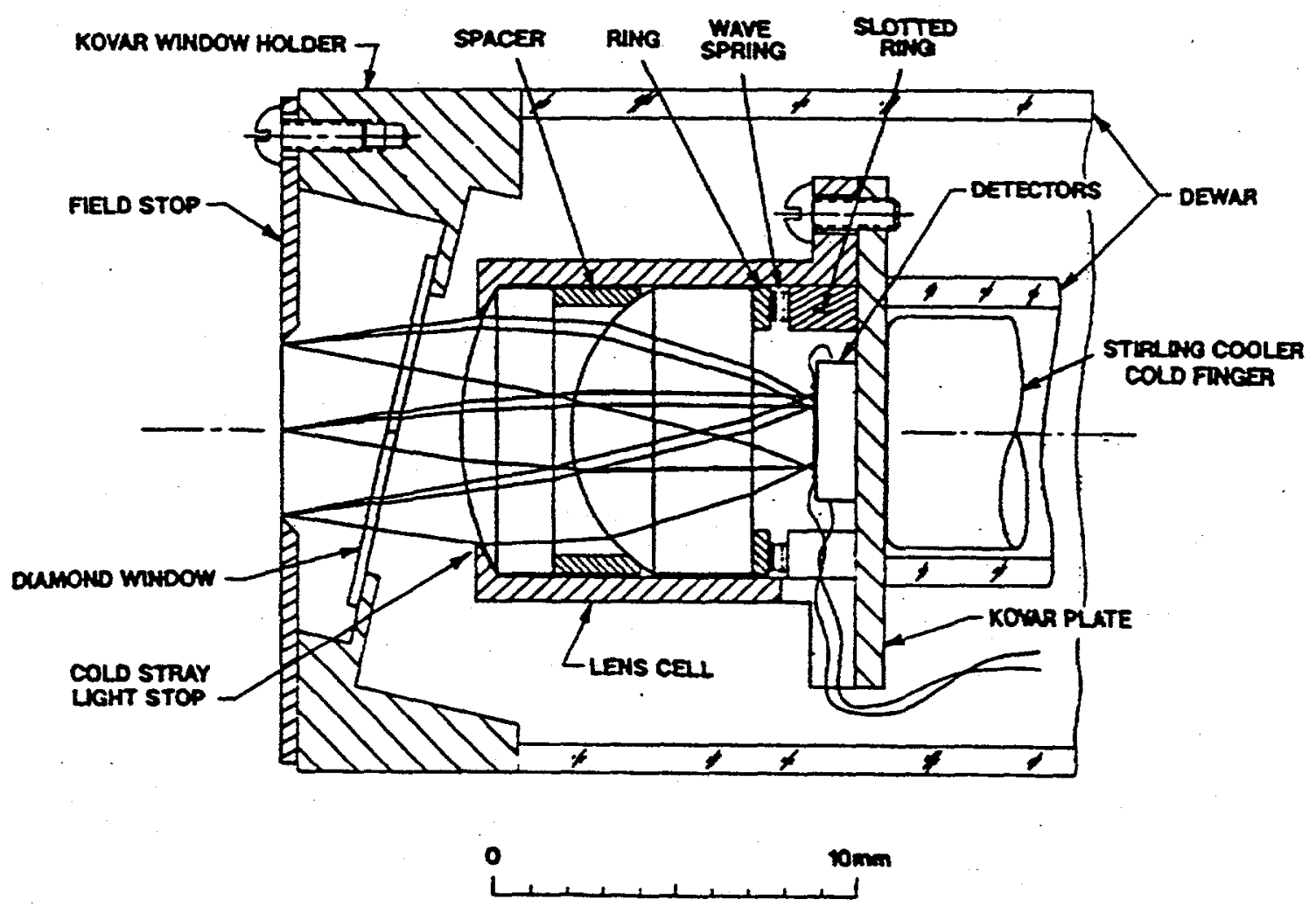

Figure 4. Small detector dewar design with focusing optics and Stirling cooler cold finger interface. 
The data is compressed onboard to reduce the data volume requirements for the real-time downlink from the UAV. Four forward and four reverse scans are co-added and each are compressed separitely. An efficient complex numerical filtering technique is applied using a TMS320C40 Digital Signal Processor (DSP). This information preserving convolution filter is followed by bit trimming for delays not close to the zero path difference. A second DSP is used for instrument control.
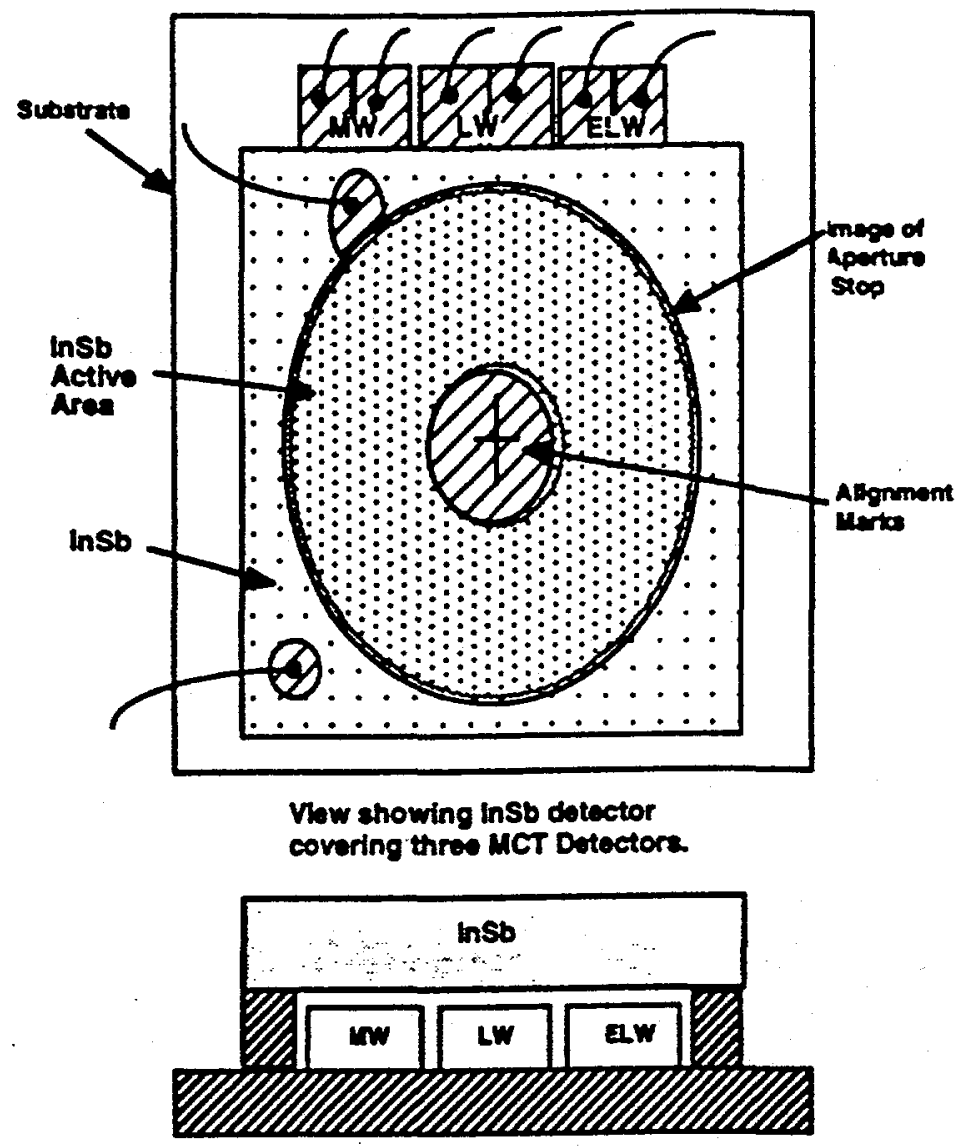

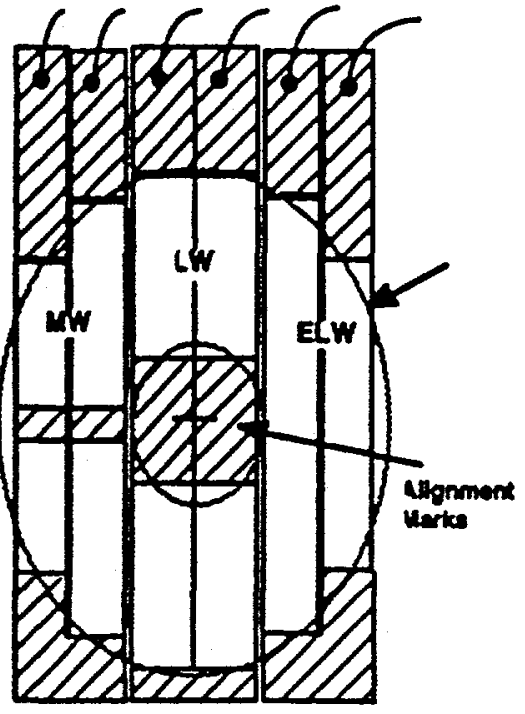

View showing three MCT defectors, all same geometry, all In the aame plane. Shaded rogions an metalized non-active arace.

Figure 5. Shared aperture focal plane design for AERI-UAV. All detectors have the same field of view to the scene, but each covers one of the four spectral bands. The configuration is a sandwich with the shortwave InSb detector suspended above the three Mercury Cadmium Telluride (MCT) detectors which share the plane illuminated by transmission through the InSb detector. The MCT detectors are each long strips, with slits down the middle to increase responsivity and to give easy lead access at one end.

\section{RADIOMETRIC PERFORMANCE}

The radiometric noise and calibration performance of the AERI-UAV is driven to a high standard both by requirements for temperature and water vapor remote sensing and for spectroscopic applications. The RMS noise level in terms of brightness temperature achieved for HIS and AERI is generally less than about $0.2 \mathrm{~K}$, except near the edges of spectral bands and in some localized spectral regions. The performance of the AERI-UAV is expected to be comparable for its 4 second dwell time, except for the extra longwave (ELW) band where longer averaging times will be needed.

The AERI-UAV will make use of the calibration techniques developed for the HIS and also demonstrated by the AERI. The calibration requirements are often stated as: <IK absolute uncertainty at a brightness temperature of $260 \mathrm{~K}$ and reproducibilities (excluding noise) of $<0.2 \mathrm{~K}$. The calibration blackbodies used by the AERI-UAV are scaled down versions ( $62 \%$ smaller due to smaller beam diameter at blackbody aperture) of those used by the AERI. These blackbodies are very well characterized and have proven to be very stable over time. Calibration techniques 
have been developed that use primary temperature and electrical resistance standards traceable to the National Institute for Standards and Technology (NIST), and a reflectance transfer standard traceable to the National Physical Laboratory. Blackbodies calibrated using these techniques have absolute temperature erors of less than $0.04 \mathrm{~K}$ peak-to-peak and an absolute emissivity error of less than 0.001 , which translates to a temperature error of $0.10 \mathrm{~K}$ peak-to-peak in the longwave band. 
Technical Digest: Optical Remote Sensing of the Atmosphere

Title of Article: Atmospheric Cooling Rate Calculations for the Airborne Southern Hemisphere Ozone Experiment (ASHOE) using MODTRAN3

Author(s): S. C. Lee, R. O. Knuteson, H. E. Revercomb, and W. L. Smith

The undersigned hereby assigns to the Optical Society of America (the "Society") copyright ownership in the above-titled work to be effective as of the date published, this assignment to be effective as of the date published and to the extend transferable under applicable national law. If each author's signature does not appear below, then the signing author(s) represent that they sign this agreement as authorized agent(s) for and on the behalf of all the authors. The Society shall have the right to register copyright to the above-titled book in its name as claimant. Also, the Society shall have the right to grant reprint permission to third parties and to negotiate and receive reasonable royalties in such instances.

Authors (or their employers, in the case of works made for hire) reserve all other rights to the above book including: (a) The right to use the work in future works of their own; (b) All proprietary rights other than copyright; (c) The right of the employer to make copies of the work. If prepared under U.S. Government contract, the govegnment shall have the rights under copyright to the extent required by the contract.

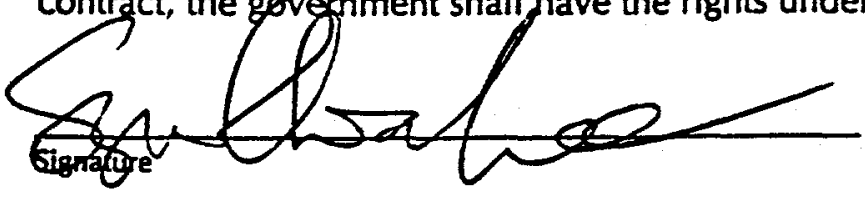

Signature

S. C. Lee

Print Name

Print Name

Research Specialis

Date

Title

Date

Government Employees-Each author who is not a U.S. Government employee should sign above, if reasonably practicable; and each author's employer should sign if the work was prepared within the scope of the author's employment.

If all authors were U.S. Government employees at the time this article was prepared, and such article was prepared as part of official duties, then one author should sign below to certify that this is true and that the article is therefore not subject to U.S. copyright protection. 
APIENDIX C: Cooling Rate Paper 
Atmospheric Cooling Rate Calculations for the Airborne Southern Hemisphere Ozone Experiment (ASHOE) using MODTRAN3

S.C. Lee, R.O. Knuteson, H.E. Revercomb, and W.L. Smith

University of Wisconsin-Madison CIMSS

1225 W. Dayton St., Madison, WI 53706

Cooling rates are derived using MODTRAN3 for ASHOE using HIS retrievals and are compared favorably with results using other global model inputs. 


\title{
Atmospheric Cooling Rate Calculations for the Airborne Southern Hemisphere Ozone Experiment (ASHOE) using MODTRAN3
}

\author{
S.C. Lee, R.O. Knuteson, H.E. Revercomb, and W.L. Smith \\ University of Wisconsin-Madison CIMSS \\ 1225 W. Dayton St., Madison, WI 53706
}

\section{Introduction}

One of the objectives of the Airborne Southern Hemisphere Ozone Experiment (ASHOE) was to examine the causes of ozone loss in the Southem Hemisphere lower stratosphere, and to investigate how the loss is related to polar, mid-latitude, and tropical processes, including radiative transfer. Cooling rates were calculated for ASHOE in order to: (1) understand how the ozone depleted air is transported to populated regions outside the polar vortex, (2) relate vertical motions and transport of air around the South Pole to atmospheric cooling rates, and (3) validate cooling rates in dynamical models for the polar vortex using observed data and more accurate radiative transfer models.

\section{Experimental Method}

This analysis follows that of Clough et al. (1992). Consider monochromatic thermal radiation for clear sky at a given level, with $\mu=$ zenith direction cosine, and $\phi$ $=$ aximuthal angle. The flux expressions may be written thus:

Upwelling flux

$$
F_{\mathrm{v}}^{+}=\int_{0}^{2 x} \int_{0}^{1} I_{\mathrm{v}}(\mu) \mu d \mu d \phi
$$

Downwelling flux

$$
F_{v}^{-}=\int_{0}^{2 x} \int_{-1}^{0} I_{v}(\mu) \mu d \mu d \phi
$$

Azimuthally integrated flux

$$
F_{v}^{ \pm}= \pm 2 \pi \int_{0}^{ \pm 1} I_{v}(\mu) \mu d \mu
$$

Three-point Gaussian quadrature was performed at the angles: $77.74^{\circ}, 53.80^{\circ}$, and $24.29^{\circ}$. This yields a net flux:

$$
F_{v}=F_{v}^{+}-F_{v}^{-}
$$

Strictly speaking, the monochromatic cooling rate is the divergence of the net flux. However, one may also express it in terms of the temperature change in a layer:

$$
\partial T /\left.\partial t\right|_{v: t,-1}=\frac{g}{C_{p}} \frac{F_{v: l}-F_{v: l-1}}{P_{l}-P_{l-1}} .
$$

\section{Results}

Cooling rate comparisons between models

In order to validate the cooling rates derived using MODTRAN3, a comparison was made to published results of Clough et al (1992). The models compared here are characterized as follows. FASCODE, a lineby-line transmittance model using HITRAN database (Clough, et al, 1986, Rothman, et al, 1992), has very high spectral resolution, but is computationally demanding. Phillips Laboratory's MODTRAN version 3 has lower spectral resolution at $2 \mathrm{~cm}^{-1}$ (Wang, et al, 1996), but is computationally fast. Calculated cooling rates between the models agree very well from $750 \mathrm{mb}$ up to $150 \mathrm{mb}$. (Fig. 1).

\section{Sensitivity study to upper level water vapor}

To assess the importance of upper tropospheric water vapor to the atmospheric cooling rates, a sensitivity study was performed using MODTRAN3. Removing the water vapor above $300 \mathrm{mb}$ affects cooling rates, not only in that region, but also in the middle troposphere (Fig. 2). Results indicate that smaller water vapor amounts cause lower cooling rates. 


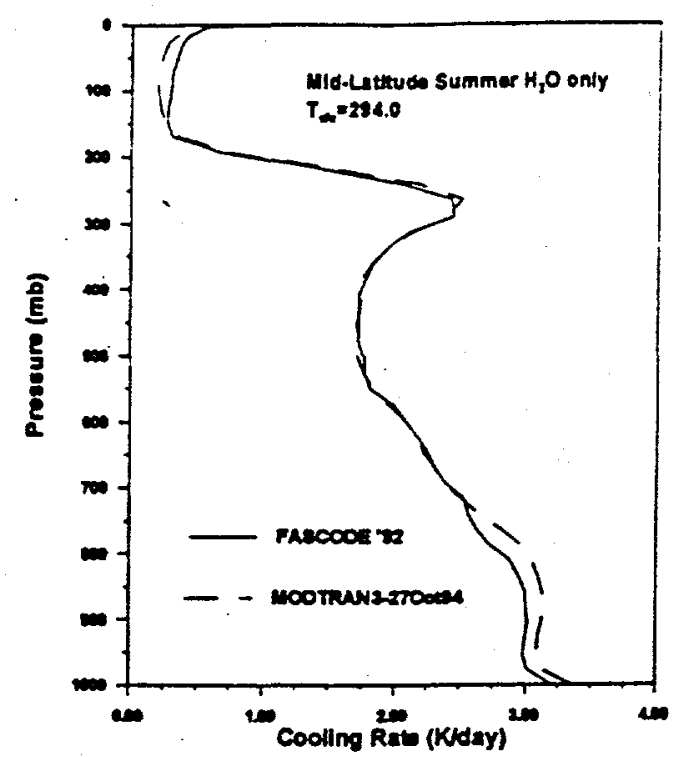

Figure 1. Cooling rate comparison

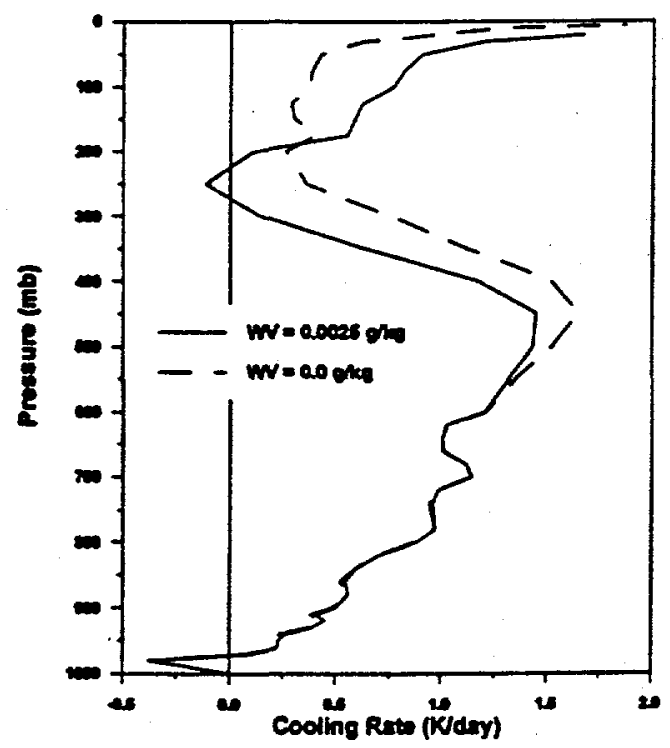

Figure 2. Comparison of cooling rates calculated with different upper atmospheric water vapor profiles

\section{Polar region cooling rates}

Retrieved temperature and water vapor from University of Wisconsin Highresolution Interferometer Sounder (HIS) (Smith et al, 1987) observations during ASHOE flights into the south polar vortex from New Zealand on 4 October 1994 were used to calculate cooling rates, which were compared to cooling rates calculated using water vapor and temperature input from four global forecast models (Fig. 3). Overall, the forecast models tend to underestimate the stratospheric water vapor concentrations in polar regions, which leads to an underestimate of the cooling rate. Figure 4 shows layer mean cooling rates from water vapor, carbon dioxide, and ozone. Water vapor contributions dominate not only in the troposphere, but also in the stratosphere. Figure 5 shows the spectral cooling rate, with contributions from the seven primary infrared optically active gases.

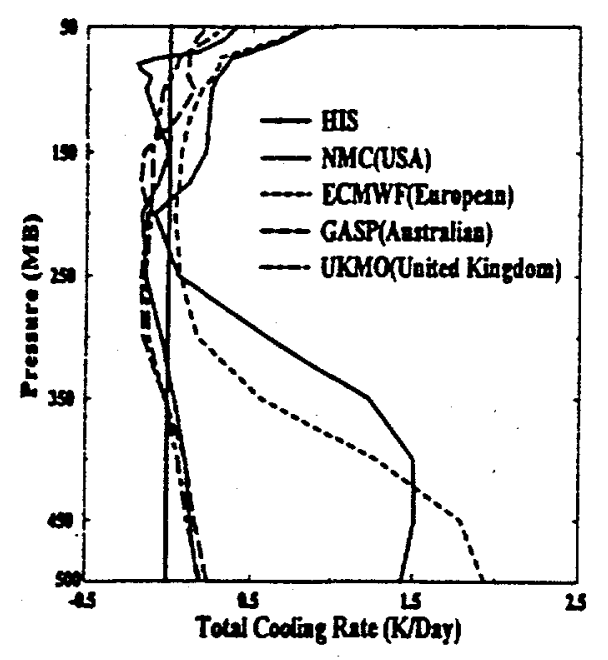

Figure 3. Cooling rate comparison for ASHOE - October $4199468 S$

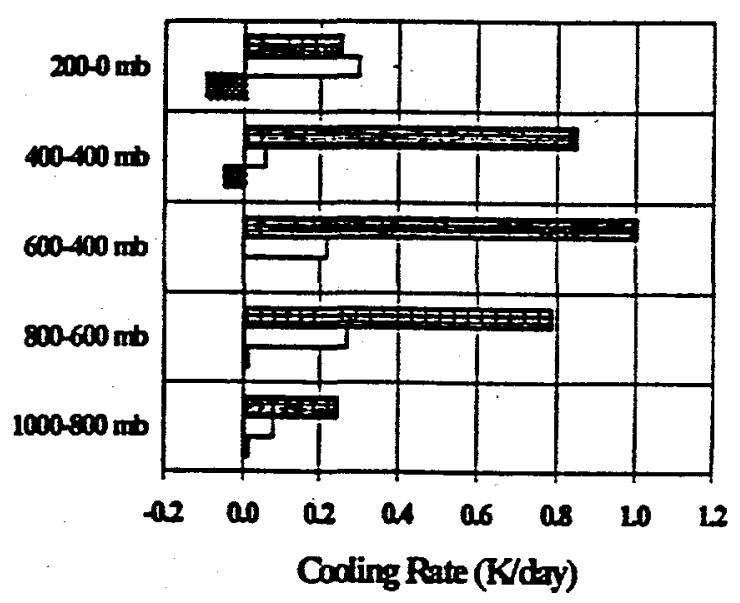

Figure 4. Layer mean cooling rates for $\mathrm{H}_{2} \mathrm{O}, \mathrm{CO}_{2}$, and $\mathrm{O}_{3}$ 


\section{Summary}

If calculation models have little or no water vapor above $200 \mathrm{mb}$, then the model will produce an error in the cooling rate of about $0.2 \mathrm{~K}$. In the upper troposphere and stratosphere, the cooling rate is not dominated by carbon dioxide alone, but also strongly by water vapor (between $400 \mathrm{~cm}^{-1}$ and $660 \mathrm{~cm}^{-1}$ ). Results of case studies show that a drier atmosphere leads to a lower cooling rate in the upper troposphere.

\section{References}

Clough, S.A., Proc. of the 6th Conf. on Atmospheric Radiation, Williamsburg, VA, 1986.
Clough, S.A., M.J. Iacono, and J.-L. Moncet, Line-by-line calculation of atmospheric fluxes and cooling rates: Application to water vapor, J. Geophys. Res., 97, 15,761-15785, 1992.

Rothman, L.S., et al, HITRAN molecular database: Edition '92, J. Quant. Spectrosc. Radiat. Transfer, 48, 469-508, 1992.

Smith, W.L., et al, 21 st International Symposium on Remote Sensing of Environment, Ann Arbor, Michigan, 1987. Wang, J., G.P. Anderson, H.E. Revercomb, R.O. Knuteson, Validation of FASCOD3 and MODTRAN3: Comparison of Model Calculations with Ground-Based and airborne Interferometer Observations Under Clear-Sky Conditions, in press, Applied Optics, 1996.

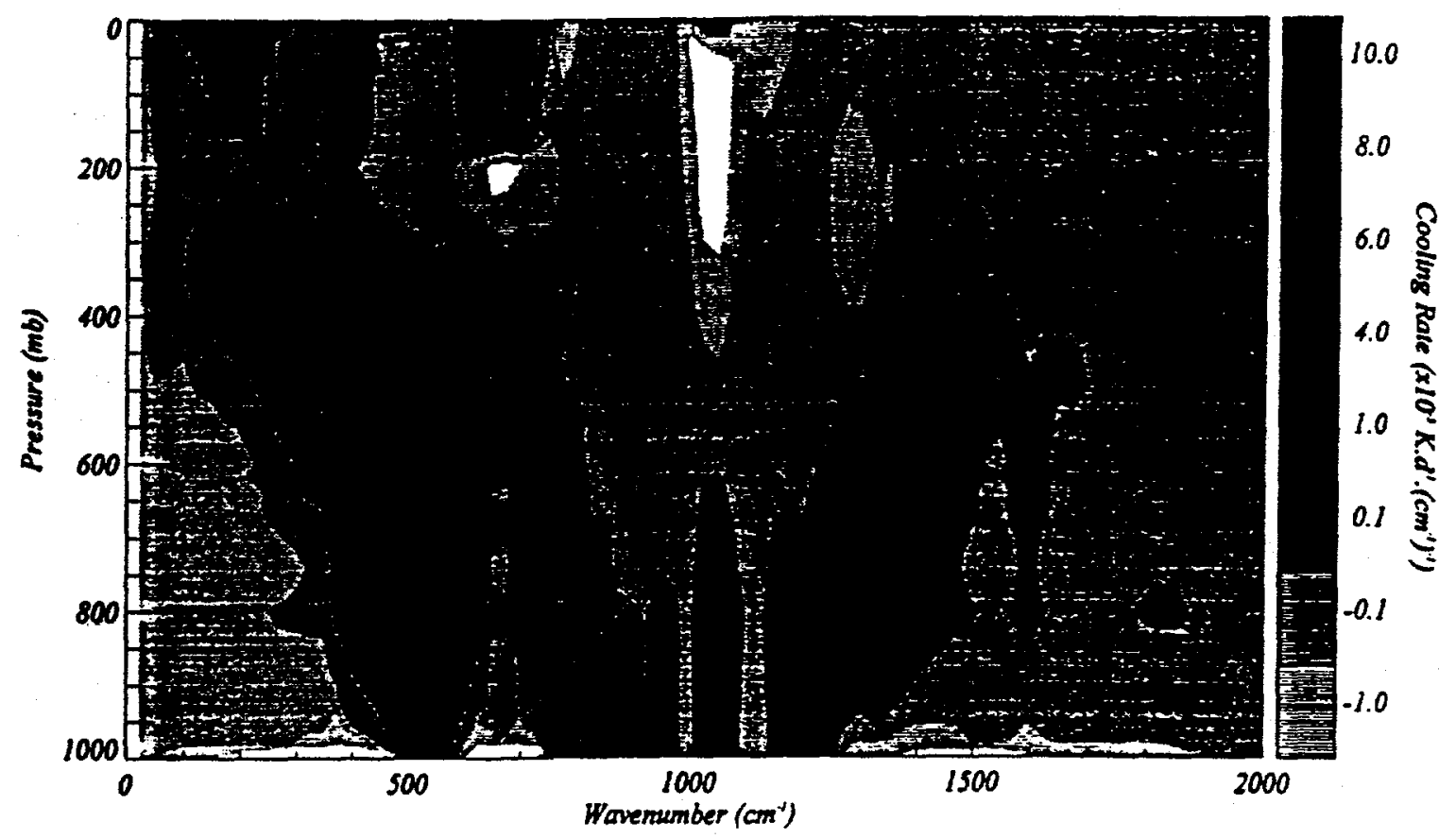

Figure 5. Spectral cooling rate profile as a linear function of pressure 